\title{
Evaluation of the Twinning Behavior of Polycrystalline Magnesium at Room Temperature by Acoustic Emission
}

\author{
Yunping $\mathrm{Li}^{*}$ and Manabu Enoki \\ Department of Materials Engineering, School of Engineering, The University of Tokyo, Tokyo 113-8656, Japan
}

\begin{abstract}
In order to study the twinning behavior of polycrystalline magnesium at room temperature, acoustic emission (AE) was measured during the compression process at an intermediate strain rate along the extrusion direction. Microstructure evolution was quantitatively characterized by the twinning area fraction and the strain dependence of twinning size distribution from the observation by optical microscope. Deformation was mainly due to twinning nucleation in the initial stage, and twinning growth and dislocation motions gradually became dominant with the increase of strain. As AE count rate increased greatly in the initial stage of deformation and dropped quickly in the later stage, AE signals in the initial stage were thought to be mainly due to the twinning nucleation. Twinning strain and the fraction of twinning strain rate were calculated by considering the variation of Schmid factor in deformation. A quantitative relation between the twinning strain and the cumulative AE counts was obtained in the initial stage of deformation. [doi:10.2320/matertrans.I-MRA2007849]
\end{abstract}

(Received September 5, 2006; Accepted February 27, 2007; Published May 25, 2007)

Keywords: pure magnesium, compression, twinning size distribution, twinning strain, acoustic emission (AE), AE count rate

\section{Introduction}

The demand of several industries such as automotive, aircraft or aerospace for a reduction of weight makes magnesium a material with high interest. ${ }^{1)}$ A low density and a high specific strength are reasons for the development of new magnesium alloys with good corrosion resistance and mechanical properties. Deformation of magnesium and its alloys typically occurs through crystallographic slipping and deformation twinning, since the uniform deformation of materials needs at least five independent slipping systems while only basal slipping system is active at room temperature, and the other systems especially twinning mainly $\{10 \overline{1} 2\}$ system have to be activated. Twinning of magnesium plays an important role in the deformation process. Twinning can lead to the reorientation of crystals and then activate other slipping systems such as non-basal slipping systems or the second order twinning system such as $\{10 \overline{1} 1\}$ system which is not active before twinning for the poor orientation direction. On the other hand, prohibiting the twinning or slip by such as decreasing the grain size (ECAE process) ${ }^{2)}$ or solution treatment (alloying) ${ }^{3)}$ can produce magnesium or its alloys with higher strength. Researches about crystallography and morphologies of the mechanical twinning in magnesium were widely conducted. ${ }^{4}$ However, the twinning behavior of magnesium with polycrystalline structure in the deformation process has seldom been studied quantitatively.

Acoustic emission (AE) is a unique technology in observing the internal dynamic behavior of microstructure evolution. ${ }^{5)}$ Twinning is one of the most important sources of AE and formation of twinning can produce much stronger elastic waves than that from the dislocation motions. ${ }^{6)}$ Some twinning or detwinning process in the martensitic transformation (MT) process can even be heard by human ears. Some AE behaviors in magnesium and its alloys have been conducted in deformation and thermal cycle. ${ }^{7,8)}$ However, no special issue has ever been focused in investigating the

*Graduate Student, The University of Tokyo quantitative relation between the twinning and the corresponding AE behavior in the deformation process.

The purpose of this paper is to study the twinning behavior of polycrystalline magnesium in an overall perspective by microstructure observation and $\mathrm{AE}$ measurement. The relation between the $\mathrm{AE}$ behavior and the twinning behavior in the compression process has been analyzed quantitatively.

\section{Experimental Procedures}

Commercial polycrystalline magnesium with purity of 99.95\% produced from Osaka Fuji Corp. was selected as the present research materials. Pure magnesium was manufactured by hot extrusion and the specimens were with the size of $\phi 15 \times 15 \mathrm{~mm}$ for compression and $100 \times 20 \times 2 \mathrm{~mm}$ for tensile specimen. All specimens were annealed at $350^{\circ} \mathrm{C}$ for 30 minutes in Ar atmosphere before deformation. Grain size is in the range of $30-50 \mu \mathrm{m}$ after heat treatment as shown in Fig. 1.

Deformation was applied parallel to the extrusion direction with a strain rate of $1.67 \times 10^{-4} / \mathrm{s}$. AE measurement was conducted in the compression process. AE system used in present research was Continuous Wave Memory (CWM) which was specially designed ${ }^{9)}$ for recording continuous $\mathrm{AE}$ signals with extremely high count rate. In the present research, AE signals were measured with a threshold of $55 \mathrm{~dB}$, and high pass filter of $100 \mathrm{kHz}$ effectively getting rid of the noises from environments. AE sensor was a low noise type (M304A, Fuji Ceramics, Japan).

Microstructure observation was conducted at different strain levels in a sequence of $0.05 \%, 0.1 \%$, and $0.2 \% \ldots$ to about $8 \%$ along the extrusion direction of sample at the center of the surface. Etching solution was aqueous solution with $5 \%$ nitric acid.

\section{Results and Discussion}

Typical deformation curves in both compression and tension along the extrusion direction are shown in Fig. 2. 


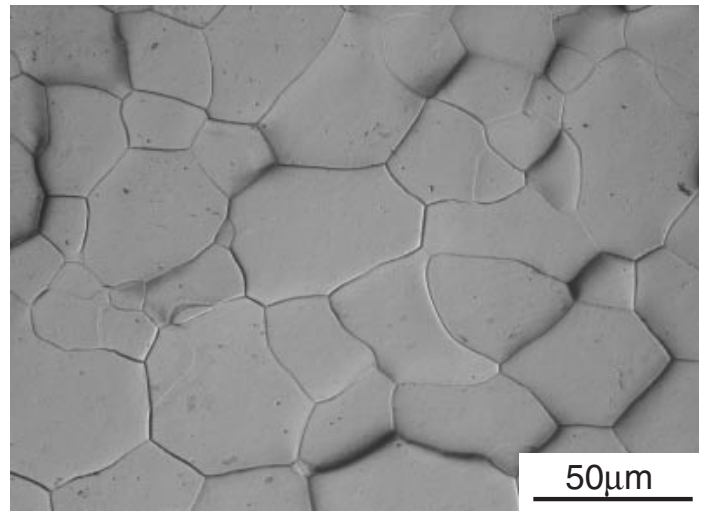

Fig. 1 Microstructure before deformation after etching by aqueous solution with $5 \%$ nitric acid.

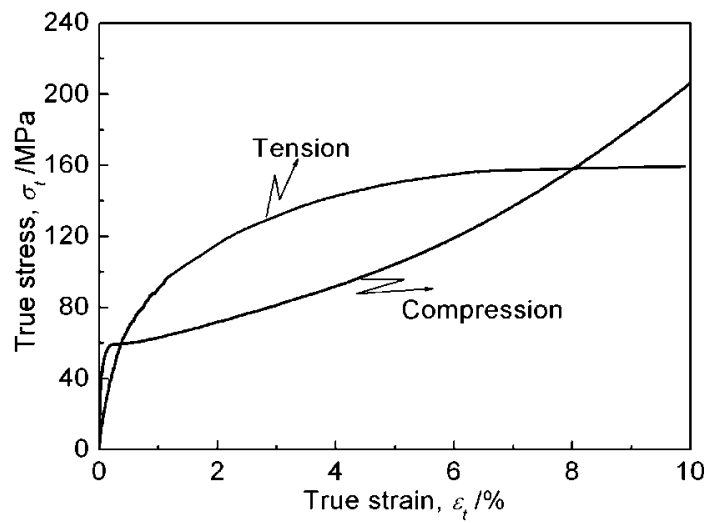

Fig. 2 Stress-strain curve of pure magnesium at room temperature shows the lower yield stress and hardening rate, and later the hardening rate increases greatly after strain of about $4 \%$ in compression.

Monotonic compression exhibits a lower yield stress and initial hardening rate than tension because $\{10 \overline{1} 2\}$ twinning system is facilitated. However, after a strain of approximately $4-5 \%$, the strain hardening rate increases rapidly for the exhaustion of twinning within the polycrystalline as well as the activation of non-basal slipping systems or the other twinning system with higher critical resolved shear stress
(CRSS) such as $\{10 \overline{1} 1\}$ system. As discussed in the previous research by X. Y. Lou et al., ${ }^{10)}$ the easily activated $\{10 \overline{1} 2\}$ twinning found in $\mathrm{Mg}$ and its alloys is tension type, i.e. one which can be activated by tension parallel to the $c$-axis in a HCP crystal structure, or equivalently, by in-plane compression. The initial crystalline texture of extruded pure magnesium exhibits a strong basal texture, where the majority of $c$ axes are aligned in the normal of extrusion direction. Present in-plane compressive stress results in twinning, while inplane tension with a contracting alone $c$-axes does not. Therefore, the compression of specimen along the extrusion direction results in a lower yield stress due to the easily activated twinning process, while the tension exhibits the normal strain-hardening aspect encountered in non-twinning materials, although some small amount of twinning is present as described in the previous research. ${ }^{10)}$ The distinctive characteristics of deformation curves in both compression and tension have been widely reported. There are three explanations that can be offered for the higher hardening rate in compression process at higher strain levels: ${ }^{11)}$

(i) twinning introduces additional barriers to dislocation movements, ${ }^{12}$ which is in some extent equivalent to reducing the grain size,

(ii) $\{10 \overline{1} 2\}$ twinning in $\mathrm{Mg}$ reorients the lattice to hard crystallographic orientations, ${ }^{13)}$ and the other twinning systems or non-basal slipping systems with the higher $\mathrm{CRSS}^{4}$ ) have to be activated,

(iii) the motions of dislocations as they pass through the twinning front can lead to the establishment of high hardening rate within the twinning interior. ${ }^{14)}$

Microstructure observation was conducted in the compressed pure magnesium as shown in Fig. 3 at strains of $0.5 \%$ and $5.0 \%$. The formation of twinning can be clearly observed and the difference in the twinning size and the twinning density at the two strain levels can be observed. Twinning size distributions at two strain levels are shown in Fig. 4. At strain of $0.3 \%$, the peak twinning length, the twinning length having the highest fraction, is about $30 \mu \mathrm{m}$, and at the strain of $2.0 \%$ is about $78 \mu \mathrm{m}$. The increase of the peak twinning length with the increase of strain level can be observed. The strain dependence of the twinning size distribution is shown in Fig. 5. Here, the contrast of the legend stands for the relative magnitude of twinning density of a specific twinning
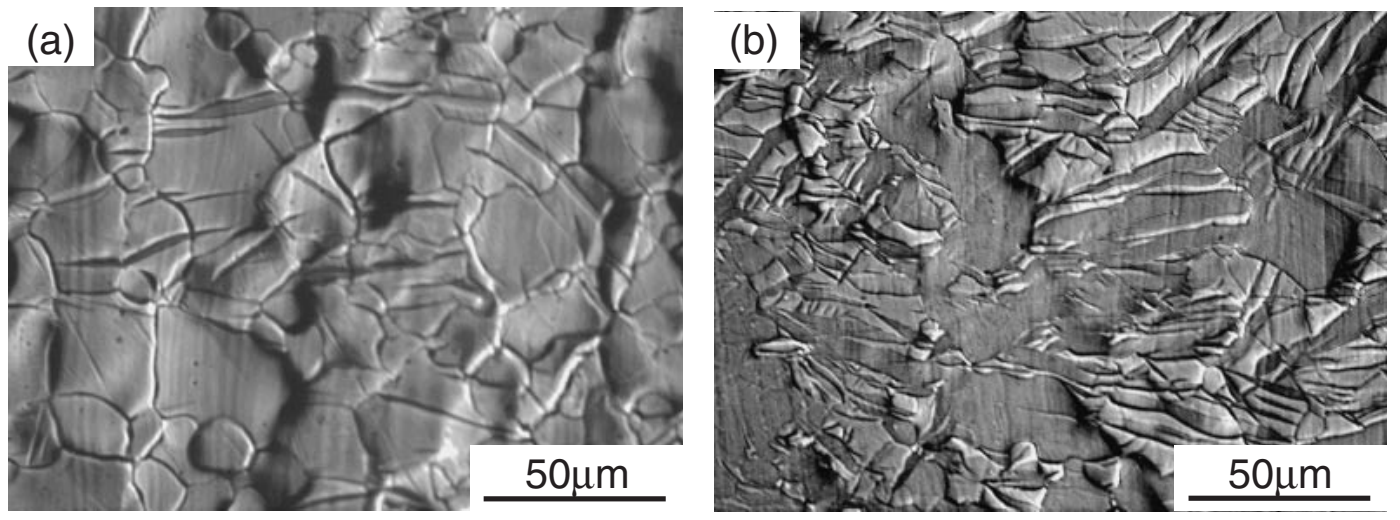

Fig. 3 Microstructure of pure magnesium after deformation at (a) strain of $0.5 \%$ and (b) strain of $5.0 \%$ shows the increase of both twinning density and twinning size with the increase of strain. 

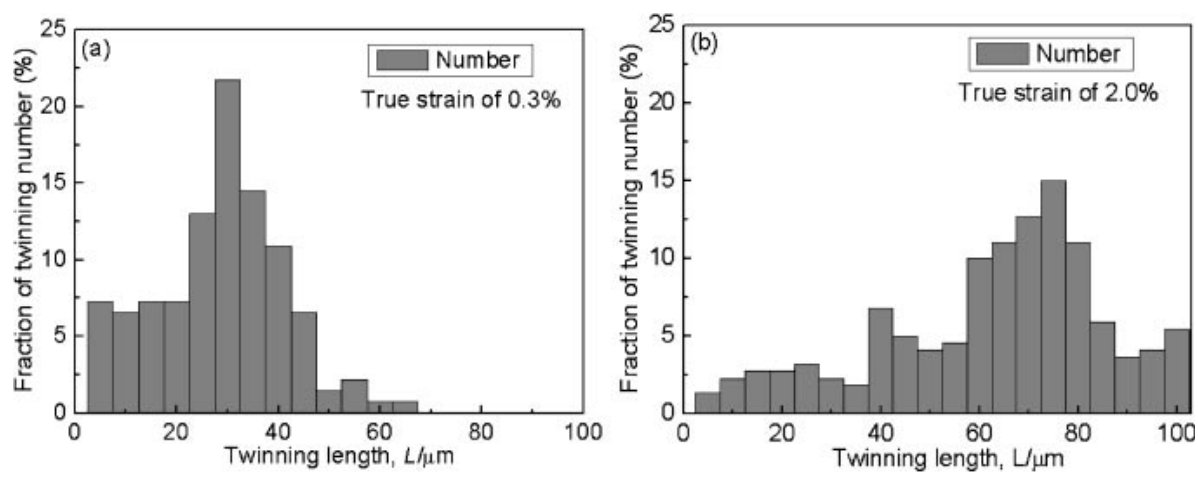

Fig. 4 Twinning size distributions at strain of (a) $0.3 \%$ and (b) $2.0 \%$ show the increase of the peak twinning length with the increase of strain.

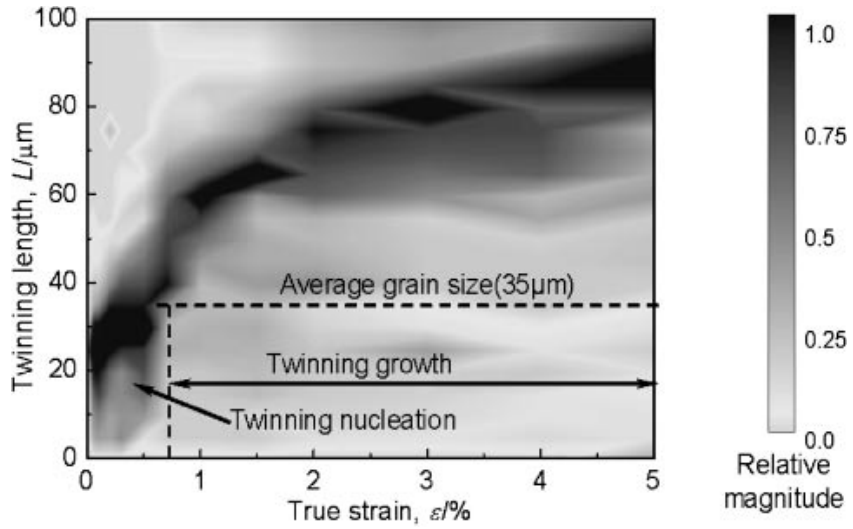

Fig. 5 Continuous distribution of twinning size for deformed pure magnesium in strain range of 0 to $5 \%$ shows the gradual increase of the peak twinning length with the increase of strain level, and before the strain level of about $0.7 \%$, the twinning are mainly formed inside of the grains.

length. As the strain level lower than about $0.7 \%$, the peak twinning length is smaller than the average grain size of materials $(35 \mu \mathrm{m})$, and later the peak twinning length greatly increases with the strain. From Fig. 5, it should be got the following conclusion that in the range of strain lower than $0.7 \%$, the twinning deformation is mainly realized by twinning nucleation and at higher strain levels is mainly realized by the twinning growth.
The short time Fourier transform (STFT) ${ }^{9)}$ of AE signals at strains of about $0.1 \%$ and $1.0 \%$ in compression are shown in Fig. 6. The X-axis, Y-axis and the contract of the legend of the STFT map are time, frequency and the relative magnitude of AE signals, respectively. At the strain of $0.1 \%$, only one frequency peak of about $490 \mathrm{kHz}$ was observed as shown in Fig. 6(a). Mainly two frequency peaks at about 200 and $490 \mathrm{kHz}$ were observed at the strain of about $1.0 \%$. The signals with lower frequency are thought to be related to the dislocation motions and the signals with higher frequency are related to the twinning formation (twinning nucleation and growth) process. It is well known that the frequency of the AE signal is determined by the rise time of AE sources. ${ }^{15)}$ The twinning nucleation with relatively higher CRSS is accompanied with a large decrease of stress and this formation velocity of twinning can exceed the transverse velocity of sound, ${ }^{16)}$ while the slipping velocity of dislocation is only about $0.3-0.4$ times of the transversal sound velocity. ${ }^{17)}$ It is thought that dislocation slipping distance is

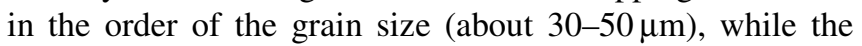
twinning nucleation distance (or the critical twinning length for twining nucleation) is smaller than that of the grain size (Fig. 5). The above characteristics results in longer rise time or lower frequency for dislocation slipping AE signals and shorter rise time or higher frequency for the twinning nucleation AE signals as schematically shown in Fig. 7 for $\mathrm{AE}$ event formation mechanism of these two sources. The
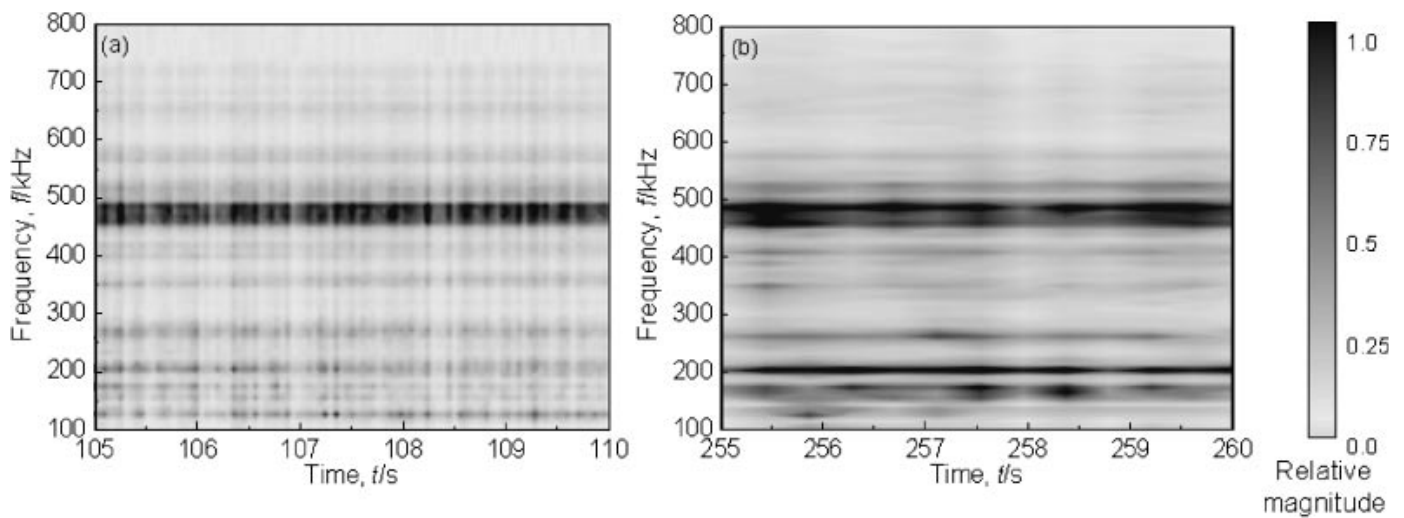

Fig. 6 STFT of AE signals in the compression process of pure Mg at strain of about (a) $0.1 \%$ and (b) $1.0 \%$ show the transition of deformation mechanism from twinning to both of the twinning and slipping. 


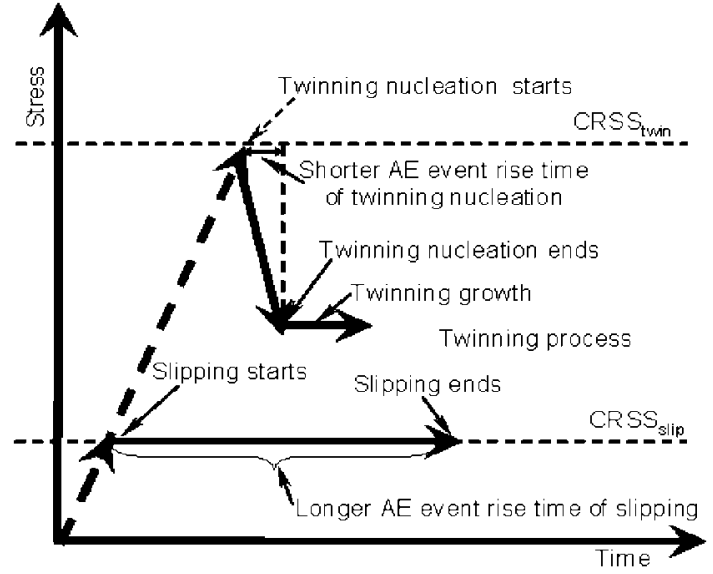

Fig. 7 The jerky formation of twinning results in a higher frequency of AE signals, and the continuous slipping produces relatively lower frequency of AE signals.

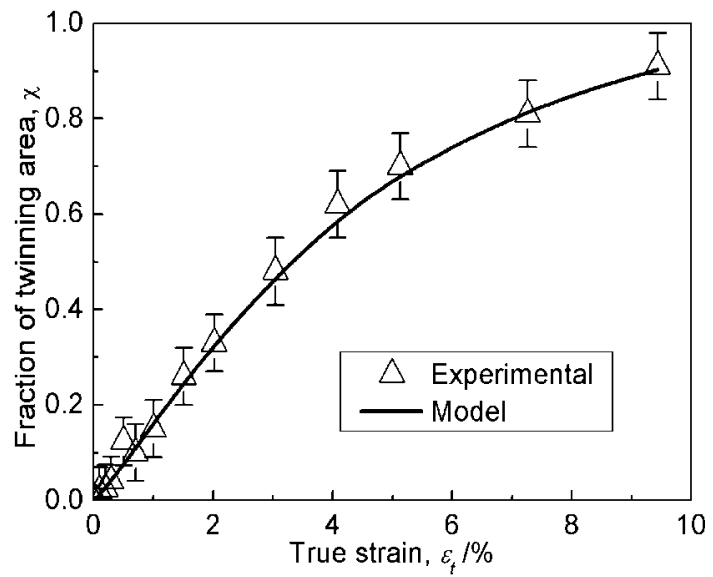

Fig. 8 Twinning area fraction of pure magnesium at different strain levels satisfies the empirical sigmoidal relation.

frequency for twinning nucleation is expected to be $2.5-3$ times of that for slipping from above analysis. It has to be mentioned that, the twinning growth and twinning nucleation are all jump-wise formation processes and are supposed to have the same frequency although the amplitude of $\mathrm{AE}$ signals in twinning nucleation is much larger than that from the twinning growth for the higher CRSS in the twinning nucleation process. ${ }^{4)}$ From Figs. 5 and 6, the following conclusion may be obtained: with the increase of strain, the deformation mechanism gradually changes from mainly twinning nucleation before the true strain of about $0.7 \%$ to the mixture of twinning formation (nucleation and growth) and slipping because the second frequency peak of about $200 \mathrm{kHz}$ becomes stronger at higher strain level as shown in Fig. 6(b).

The twinning density as a function of true strain level described by twinning area fraction is shown in Fig. 8. The twinning density increased gradually with the increase of the strain levels and the fraction of twinning area in the deformation of $\mathrm{Mg}$ is followed by the sigmoidal equation, ${ }^{18}$ )

$$
\chi=1-\exp \left[-\gamma\left(\varepsilon / \varepsilon_{0}\right)^{\beta}\right]
$$

where the value of $\varepsilon_{0}$ is theoretical strain when the twinning area fraction is unity, and $\beta$ is the rate exponent. The value of $\varepsilon_{0}$ is necessarily greater than the true strain $\varepsilon$. The nonlinear fit result by selecting appropriate value of $\beta, \varepsilon_{0}$ and $\gamma(1.16$, 0.15 and 4 , respectively) shows a good agreement between the experimental data and the nonlinear fit result as shown in Fig. 8. Twinning area fraction strain rate can also be obtained by a differential application in $\varepsilon$ from eq. (1),

$$
\frac{d \chi}{d \varepsilon}=\frac{\gamma \beta}{\varepsilon_{0}}\left(\varepsilon / \varepsilon_{0}\right)^{\beta-1} \exp \left[-\gamma\left(\varepsilon / \varepsilon_{0}\right)^{\beta}\right] .
$$

Obviously, twinning plays an important role in the deformation process of pure $\mathrm{Mg}$. It is interesting to consider how much of the strain is accommodated by twinning. For a given grain orientation, $\varepsilon_{t w}$ is the strain accommodated by twinning that is thought to be related to the characteristic shear of twinning system $S$, the Schmid factor $m$ supposed to be decreased with the increase of strain $\varepsilon$, as well as the volume fraction of the twinned grain $v^{10)}$ by

$$
\varepsilon_{t w}=m v S \text {. }
$$

In the in-plane compression process, the initial Schmid factor of extruded $\mathrm{Mg} m_{i}$ is found to be approximately $0.32,{ }^{19)}$ and it is proposed that the Schmid factor $m$ decreases linearly to the true strain,

$$
m=M m_{i}, M=1-C \varepsilon,
$$

where $C$ is a constant of $10 / 3$, since it is supposed that the Schmid factor will decrease to the half of the initial value at the strain $\varepsilon_{0}(=0.15)$. Assuming the volume fraction of twinning $v$ is approximately equal to the area fraction, since there is a large variance in measuring the twinning density. Then the strain accommodated by twinning may be written as

$$
\varepsilon_{t w}=m S \chi=m_{i} S M \chi,
$$

The fraction of twinning strain rate, the increase of twinning strain per overall strain, can be written by following equation by a differential in true strain $\varepsilon$ from eq. (5),

$$
\frac{d \varepsilon_{t w}}{d \varepsilon}=m_{i} S\left(\chi \frac{d M}{d \varepsilon}+M \frac{d \chi}{d \varepsilon}\right)
$$

where $d M / d \varepsilon$ is $10 / 3, \chi$ and $d \chi / d \varepsilon$ can be obtained from eqs. (1) and (2), respectively. Equations (5) and (6) demonstrate more precise equations than previous research since the reorientation of the texture in deformation has been considered. ${ }^{20)}$ Using eqs. (5) and (6) and the shear of tension twinning $\{10 \overline{1} 2\}$ in pure $\mathrm{Mg}, 0.131$, we estimated the twinning strain and the fraction of twinning strain rate along with the true strain in the strain range of 0 to $8 \%$ as shown in Fig. 9. It has to be mentioned that in the initial stage of deformation, the twinning strain rate is not the highest due to the large fraction of elastic deformation (about $40 \%$ in present calculated result), and with the increase of strain, the strain rate attributed by twinning will attain a peak value and then decrease gradually. The AE results in Fig. 9 show that the $\mathrm{AE}$ count rate is related to the fraction of twinning strain rate closely, and in the deformation of pure $\mathrm{Mg}$, the $\mathrm{AE}$ signals are mostly attributed by the twinning process. At higher strain levels (strain $>4 \%$ ), the second and the third peak of the $\mathrm{AE}$ count rate are thought to be related to the 


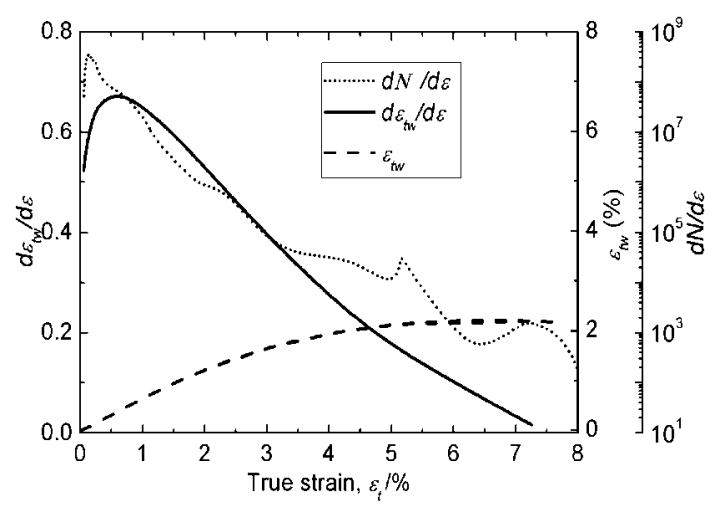

Fig. 9 Calculated fraction of twinning strain rate and twinning strain by eqs. (5) and (6), true strain dependence of $\mathrm{AE}$ count rate showing $\mathrm{AE}$ count rate strongly related to the twinning strain rate fraction before true strain of $4 \%$.

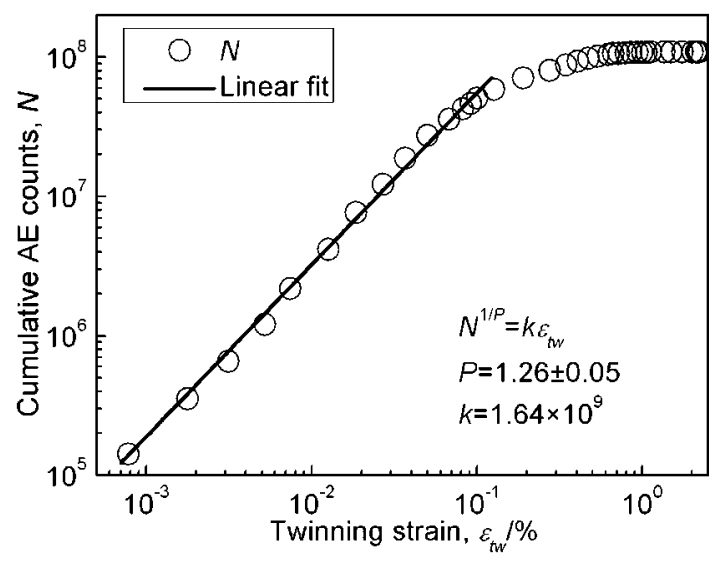

Fig. 10 Twinning strain dependence of cumulative AE counts $N$ shows an exponential relation between twinning strain and cumulative AE counts.

activation of other twinning systems such as $\{10 \overline{1} 1\}$ twinning as discussed previously. ${ }^{4)}$

The calculated twinning strain and the corresponding cumulative AE counts in compression are plotted in Fig. 10. It is found that the relationship between cumulative $\mathrm{AE}$ counts and the twinning strain can be expressed by following relation in the initial stage of deformation process,

$$
N^{1 / P}=k \varepsilon_{t w},
$$

where $P$ is an exponent constant with a value of about 1.26 in present situation, and $k$ is about $1.64 \times 10^{9}$. With the increase of twinning strain (twinning strain larger than $0.1 \%$ ), the relation between cumulative $\mathrm{AE}$ counts and twinning strain can not be expressed by eq. (7) any more because the deformation mechanism of pure magnesium transferred from the twinning nucleation to the twinning growth and slipping accordingly.

$\mathrm{AE}$ is the radiation of stress waves by materials in which local dynamic restructuring of the internal structure occurs with the release of elastic energy. The dislocation group motions with very high speed will release elastic energy. Twinning nucleation and growth with extremely high speed also produces high elastic energy. Twinning is especially important in crystals of magnesium with low symmetry where the five independent slipping systems to satisfy the criterion for a general deformation may not be available. So, AE signals in the deformation process consist of both the information from dislocation motions and the twinning process basically. However, for magnesium, the CRSS of $\{10 \overline{1} 2\}$ twinning formation (nucleation and growth) is higher than that from basal slipping, ${ }^{4)}$ and as shown in Fig. 7 the elastic energy per event released in basal slipping system should be lower than that from twinning because there is a large decrease of applied stress in twinning formation. So, the detected AE signals should be mainly from twinning nucleation in the initial stage of deformation and from twining growth and the dislocation motions at higher strain level as shown in the STFT results that two frequency peaks were observed at strain of $1.0 \%$ and only one peak at strain of $0.1 \%$. The strain dependence of twinning length distribution and the exponential relation between cumulative AE counts and the twinning strain also exhibit that the extremely high $\mathrm{AE}$ count rate in the initial stage of deformation was mainly due to the twinning nucleation, and later the relative weak $\mathrm{AE}$ signals with low AE count rate were mainly resulted from the dislocation motion and twinning growth.

\section{Conclusions}

(1) The distinguished deformation behaviors of pure magnesium in both compression and tension were due to the twinning in the compression which results in a lower yield stress and hardening rate in initial stage of compression process as well as the higher strain hardening rate at higher strain levels.

(2) The strain dependence of twinning length distribution showed that before the strain of about $0.7 \%$, the deformation was mainly accommodated by the twinning nucleation and accommodated by the twinning growth at higher strain levels.

(3) Twinning strain and fraction of twinning strain rate were calculated by considering the variation of Schmid factor in the deformation process of textured structure and the results showed that the AE count rate and the fraction of twinning strain rate demonstrate the similar behaviors.

(4) Exponential relation between twinning strain and cumulative AE counts was obtained in the initial stage of compression process. This relation approximately demonstrates the relation between the cumulative $\mathrm{AE}$ counts and twinning strain accommodated by twinning nucleation.

\section{REFERENCES}

1) B. L. Mordike and T. Ebert: Mater. Sci. Eng. A 302 (2001) 37-45.

2) J. Koike and T. Kobayashi: Acta Mater. 51 (2003) 2055-2065.

3) Y. Wang, G. Liu and Z. Fan: Acta Mater. 54 (2006) 689-699.

4) J. W. Christian and S. Mahajan: Prog. Mater. Sci. 39 (1995) 1-157.

5) R. K. Miller: Nondestructive testing handbook, second edition, volume 5, AE testing, (American society for nondestructive testing, 1987) pp. $1-50$.

6) K. Florian, B. Thomas, D. Gerhard, W. Alexander and C. Helnut: Intermetallics 8 (2000) 823-830.

7) J. Bohlen and F. Chmelík: J. Alloy. Comp. 378 (2004) 214-219. 
8) C. Frantsek and L. Pavel: Mater. Sci. Eng. A 325 (2002) 320-323.

9) K. Ito and M. Enoki: Mater. Trans. 48 (2007) 1221-1226.

10) X. Y. Lou, M. Li, R. K. Boger, S. R. Agnew and R. H. Wagoner: Int. J. Plasticity 23 (2007) 44-86.

11) M. R. Barnett, Z. Keshavarz, A. G. Beer and D. Atwell: Acta Mater. 52 (2004) 5093-5103.

12) I. Karaman, et al.: Acta Mater. 48 (2000) 2031-2047.

13) S. R. Agnew, M. H. Yoo and C. N. Tome: Acta Mater. 49 (2001) 42774289.

14) Z. S. Basinski, et al:: Acta Mater. 49 (2001) 3097-3108.
15) W. Schaarwachter and H. Ebener: Acta Metall. Mater. 38 (1990) 195205.

16) P. Gumbsch and H. J. Gao: Science 283 (1999) 965-968.

17) J. Friedel: Dislocation, (Pergamon press, New York, 1964) pp. 55-96.

18) M. R. Barnett, C. H. J. Davies and X. Ma: Scr. Mater. 52 (2005) 627632.

19) D. W. Brown and S. R. Agnew: Mater. Sci. Eng. A 399 (2005) 1-12.

20) S. Kleiner and P. J. Uggowitzer: Mater. Sci. Eng. A 379 (2004) 258262. 